\title{
Chapter 8 \\ Acute and Chronic Infection \\ Management in CF
}

\author{
Patrick Allen, Joseph Borick, and Jamie Borick
}

\section{Pathophysiological Basis for Infection}

The cystic fibrosis transmembrane conductance regulator (CFTR) protein regulates salt and water balance on epithelial cells. In the lungs, the loss of function of the CF transmembrane conductance regulator decreases the hydration of the airways through multiple mechanisms including creation of a hyper-absorptive state [1]. Without proper mucus hydration there is less mucociliary clearance of bacteria, leading to gross retention of pathogens [1]. Pathogens such as Pseudomonas aeruginosa, Staphylococcus aureus, Burkholderia cepacia, Achromobacter, Stenotrophomonas, and Mycobacteria often become colonized in the lower airways. This leads to chronic inflammation and irreversible tissue damage of the airways. Severe bronchiectasis is the end result, sheltering niduses of pathogen-laden mucus, which become increasingly difficult to clear. At times, acute exacerbations of these chronic infections can occur, leading to the temporary worsening of already compromised lung function.

CF patients may harbor multiple coexisting microcolonies with distinct mixtures of pathogens in separate lung loci. Therefore, sputum cultures may not be indicative of the total pathogen burden, and reliance on a single culture may obscure the presence of unique bacterial species, strains, and resistance profiles [2]. This pathogen diversity increases the difficulty of targeting appropriate and specific antimicrobial therapy during an acute pulmonary exacerbation.

\footnotetext{
P. Allen $(\varangle) \cdot$ J. Borick

Department of Family Medicine, University of Kansas SOM Wichita, Wichita, KS, USA

Via Christi Family Medicine Residency, Wichita, KS, USA

e-mail: Patrick.Allen@ ascension.org; JBorick@kumc.edu

J. Borick

Department of Internal Medicine and Pediatrics, University of Kansas SOM Wichita,

Wichita, KS, USA

e-mail: JBorick2@kumc.edu
} 


\section{Epidemiology}

Cystic fibrosis is the most common life-threatening inherited disorder in people of European decent with a 1 in 25 carrier rate [3]. Some have hypothesized that a heterozygote advantage must exist for CF carriers, selecting for such a high prevalence of the allele among Caucasians. One theory is that partially impaired CFTR function in heterozygous carriers may mitigate the severity of certain life-threatening infections such as cholera and increase survival [4]. However, homozygous individuals, with a double inheritance of CFTR gene mutations, suffer from chronic infectious complications which come with the cystic fibrosis disease.

Over the last 30 years, the single overall most common respiratory pathogen affecting CF patients has changed from P. aeruginosa to S. aureus (Fig. 8.1) [5]. However, many other microorganisms are also implicated, and their relative distributions shift greatly based on patient age. While $P$. aeruginosa is still the most common pathogen isolated from older adults with $\mathrm{CF}$, children and younger adults reflect the overall trend and are now more likely to culture $S$. aureus from their sputum (Fig. 8.2) [5].

While $S$. aureus and $P$. aeruginosa are the most common pathogens in $\mathrm{CF}$ patients, other organisms such as Burkholderia cepacia, Achromobacter, Stenotrophomonas, nontuberculous mycobacteria, and anaerobes can also be associated with worsening lung function and increased mortality. Patterns will vary regionally and between different $\mathrm{CF}$ centers.

\section{Prevalence of Respiratory Microorganisms, 1991-2017}

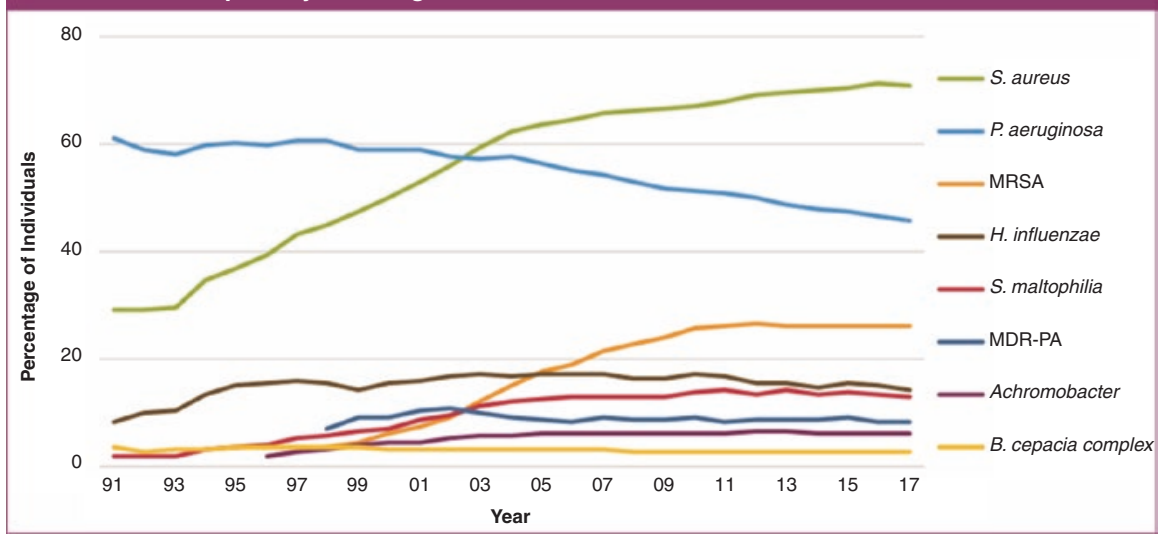

Fig. 8.1 CF Respiratory Pathogens over Time 
Prevalence of Respiratory Microorganisms by Age Cohort, 2017

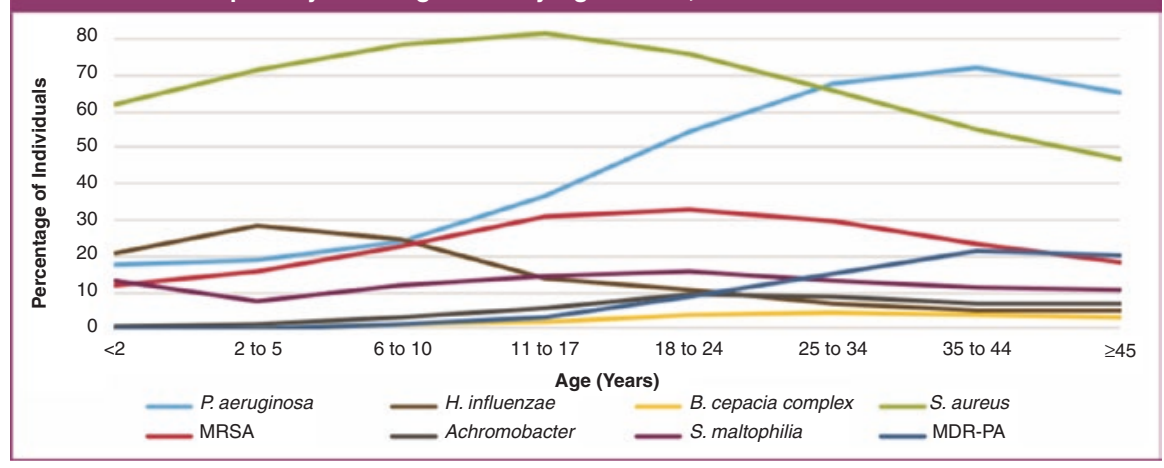

Fig. 8.2 CF Respiratory Pathogens by Age

\section{Clinical Microbiology in Cystic Fibrosis}

\section{Staphylococcus aureus $(S A)$}

SA is a gram-positive coccus which has become a ubiquitous colonizer and pathogen in healthcare. It is found in about $48 \%$ of oropharyngeal cultures in healthy US children [6].

SA is even more common in the sputum samples of patients with $\mathrm{CF}$ than in the general population. In 2011 the prevalence reached its peak at $68 \%$ and has leveled off at just over half of isolates since then [5].

However, the prevalence specifically of methicillin-resistant S. aureus (MRSA) continues to increase. As of $2017,25 \%$ of CF patients had positive sputum cultures for MRSA [5]. This is highly concerning as MRSA-positive sputum cultures are associated with worse pulmonary function as well as increased mortality compared to patients without MRSA [7]. There is also evidence for pathogen synergy. For example, PA produces more virulence factors such as pyocyanin in the presence of SA [1].

\section{Pseudomonas aeruginosa $(P A)$}

PA is a gram-negative rod, which, though rarely found in healthy individuals, is ubiquitous in the environment [5]. It is a common and serious pathogen in $\mathrm{CF}$ patients, leading to both reduced lung function and increased mortality [8]. Historical emergence of PA as a major pathogen in CF centers is part of what led to the development of the Infection Prevention and Control Clinical Care Guidelines [9]. The sputum prevalence of PA increases with age from rates of less than $20 \%$ in 5 -yearolds to $70 \%$ or more by the age of 30 [5]. PA thus becomes a major cause of pulmonary exacerbations in adult patients. 
PA is incredibly adaptable. De novo mutations are commonplace for PA; while the initial clone may remain the most clinically apparent strain for many years, phenotypically distinct strains can develop hidden within segregated microcolonies [10]. PA virulence factors can also change based on interactions with the host or other bacteria. One example is PA's increased production of pyocyanin when exposed to some gram-positive bacteria [1].

PA seems able to adapt specifically to the unique environment of CF lungs. Lung epithelial cells secrete a relative lack of glutathione in CF patients, causing an oxidatively stressful environment. PA responds by morphing into a mucoid variant and hypersecreting a mucoid polysaccharide called alginate. This forms the matrix for a biofilm, a structured consortium of bacteria and polymers. Biofilms shield against antimicrobials as well as host immune defenses such as phagocytosis. They become quite proinflammatory, triggering host humoral and cellular pathways which ultimately lead to lung tissue destruction [11].

To evade the effects of antibiotics, PA can slow its doubling time and thus its susceptibility to them. PA can also directly produce toxins, and strains may employ an impressive number of antibiotic resistance mechanisms such as efflux pumps. The sheer virulence of PA mandates that its continued presence be considered a chronic infection rather than simply colonization [11].

PA infection is often classified by the Leeds Criteria, based on PA in sputum cultures from the previous year. Patients are classified in one of four groups [12]:

- Never infected: PA has never been cultured.

- Free of PA: no growth over the previous 12 months.

- Intermittent: $<$ or $=50 \%$ of previous 12 months had PA growth.

- Chronic: $>50 \%$ cultures positive for PA in last 12 months.

These categories have been correlated with clinical outcomes - the "never" and "free" groups experiencing the least sequelae and the "chronic" group experiencing the most [12].

Fortunately, the prevalence of PA in CF sputum cultures has consistently decreased over the last 20 years. In 1997, 40\% of children had positive sputum cultures for PA. In 2017, this number decreased to $27 \%$ [5]. This is likely due to increasing efforts to eradicate PA after the first positive sputum culture [13].

While the rates of positive sputum cultures for PA have decreased over time, the rates of multidrug resistant (MDR) PA remain unchanged. As the CF population ages and sustains more cumulative exposure to antibiotics, more antibiotic resistance emerges [5].

\section{Burkholderia cepacia Complex}

Burkholderia cepacia complex (BCC) is a group of gram-negative rods which can cause a particularly devastating clinical course in patients with CF. Prior to 1992 Burkholderia spp. were included in the Pseudomonas genus. BCC can survive in 
many environments and has even been found in disinfectants, bronchoscopy equipment, urinary catheter equipment, and ultrasound gel [14]. BCC was present in $2.4 \%$ of CF patients in 2017 [5].

In the immunocompetent host, $\mathrm{BCC}$ is relatively avirulent. However, in $\mathrm{CF}$ patients its presence conveys worse clinical outcomes. When compared with PA infection, $\mathrm{BCC}$ is associated with a more rapid decline in BMI, and B. cenocepacia, in particular, is associated with a more aggressive decline in FEV1 [15]. Burkholderia cenocepacia and B. multivorans are the most common strains isolated from $\mathrm{CF}$ patients [1]. Classically, B. cenocepacia has been associated with a more rapid decline in FEV1 than B. multivorans [16]. If B. cepacia is isolated, it should be sent to the CF reference library at the University of Michigan [17].

Some patients with BCC develop a life-threatening, sometimes necrotizing, pneumonia, associated with bacteremia and severe respiratory failure. This often fatal clinical picture is referred to as cepacia syndrome and is one of the most feared infectious complications in CF care [15]. Antibiotic resistance commonly complicates treatment.

\section{Nontuberculous Mycobacteria}

Nontuberculous mycobacteria (NTM) are acid-fast bacilli that are ubiquitous in the environment. They are commonly found in the soil as well as water sources. Human infection is usually due to exposure to NTM in the environment rather than nosocomial or person to person spread [18].

NTM's prevalence is on the rise in both CF patients and the general population [5]. In patients with CF, NTM sputum positivity has increased from $1.3 \%$ in 1984 to $13 \%$ in 2017 [19]. Patients with NTM suffer from a more rapid decline in pulmonary function than control patients without NTM although coinfections with SA and Aspergillus may be to blame [20]. Compared to Mycobacterium avium complex, Mycobacterium abscessus complex (MABSC) is more difficult to eradicate and more likely to lead to death or lung transplantation [21]. These two species make up 95\% of the NTM seen in patients with CF [22]. Unfortunately, MABSC has increased from $16 \%$ of NTM in 2003 to $42 \%$ in 2017 [18].

\section{Stenotrophomonas maltophilia}

Stenotrophomonas maltophilia is a gram-negative rod found in up to $30 \%$ of young adult CF patients [23]. While it is becoming a more recognized organism, the degree of its pathogenicity is debated. Some studies have found a threefold increase in death or lung transplant in CF patients chronically infected with S. maltophilia [24]. However, other studies have attributed this observation to confounding factors such as pancreatic insufficiency, number of hospitalizations, and coinfection with PA or BCC [25]. Another study showed that a serologic response to S. maltophilia was 
indeed independently associated with pulmonary exacerbations, suggesting a complex role that the host response may have on clinical disease [26].

\section{Achromobacter xylosoxidans}

Achromobacter xylosoxidans is a gram-negative bacillus that inhabits water and soil. It was found in $5.7 \%$ of CF patients in 2017 [5]. The clinical impact of $A$. xylosoxidans is not well defined at this time. Chronic infection seems to induce inflammation and an FEV1 decline similar to patients chronically infected with PA [27]. In a subset of chronically infected patients with high Achromobacter-specific antibodies, FEV1 decline was worse still [28]. However, there are also multiple studies that have found no significant difference in BMI or FEV1 in CF patients with A. xylosoxidans $[29,30]$.

\section{Other Bacteria}

The microbiome of CF patients extends far beyond the bacteria described above, and loss of microbial biodiversity is associated with worse lung function [31]. Other bacteria such as Haemophilus influenzae, anaerobes (like Streptococcus milleri, Prevotella, Actinomyces, and Veillonella), and newer bacteria such as Gemella and Rothia mucilaginosa are also common pathogens [32]. Anaerobes are especially interesting as they survive well in mucoid surfaces and biofilms and are often associated with worse outcomes [1].

\section{Fungi}

Aspergillus fumigatus, Candida albicans, and Scedosporium apiospermum are the most commonly isolated fungi in CF [33]. Due to the inability to effectively clear fungi from the lower respiratory tract, $\mathrm{CF}$ patients are often colonized by the above organisms causing chronic exposure to fungal antigens [34]. Rather than causing invasive pulmonary disease, fungi may cause type I or type III hypersensitivity reactions with increased eosinophilia and IgE due to a TH2 response [34].

The most commonly studied fungus in CF patients is Aspergillus. The prevalence of Aspergillus spp. in sputum cultures of CF patients increases with age as well as with worsening lung function. On average Aspergillus colonizes $39 \%$ of CF patients with a prevalence ranging from $6 \%$ in children to up to $58 \%$ in adults [35-37].

It is unclear whether Aspergillus colonization is associated with adverse outcomes. However, the development of allergic sensitivity against Aspergillus has been associated with worsening FEV1 and more frequent pulmonary exacerbations [38]. This allergic bronchopulmonary aspergillosis (ABPA) is a clinical phenomenon which occurs in just $1-15 \%$ of CF patients and is not unique to CF [37]. ABPA 
is characterized by clinical worsening with cough, wheezing, and decline in pulmonary function without another known cause. Diagnosis of ABPA requires elevated total IgE levels and evidence of allergic sensitization to Aspergillus (either positive specific IgE or positive skin prick test). Other findings may include anti-Aspergillus IgG, peripheral eosinophilia, or chest imaging [39]. The goal for management of ABPA is to decrease the inflammation and eventual fibrosis that occur due to hypersensitivity towards the continued presence of the fungal antigens.

Candida species are commonly found in sputum cultures from CF patients with a prevalence as high as 75\% [40]. Chronic colonization with Candida has been associated with worse outcomes in some studies but not others [38, 41]. Whether any negative clinical effects are attributable to Candida colonization remains unclear. Candida colonization is more likely in patients with diabetes, and in patients who use chronic inhaled antibiotics or steroids. Patients are also commonly coinfected with infectious pathogens. These confounders likely have more of an effect on poor lung function than colonization with Candida itself. Furthermore, in contrast to Aspergillus, allergic sensitization against Candida does not seem to cause lung function decline and pulmonary exacerbations [38].

Other fungi often colonize the respiratory tract in CF patients. Scedosporium colonizes up to $10 \%$ of CF patients and is usually nonvirulent. However, posttransplant immunosuppression can lead to pathogenicity, and Scedosporium can become invasive, disseminated, and potentially deadly [42]. It is unfortunately resistant to many antifungals such as amphotericin B [42]. Voriconazole, posaconazole, and ravuconazole are the drugs of choice and may even be used in combination with terbinafine if needed [42].

Exophiala dermatitidis is a common colonizer in CF patients. It is more frequently found in adults and preferentially colonizes patients with pancreatic insufficiency or colonization with Aspergillus or NTM [43]. While there are case reports of disseminated disease in non-CF immunocompromised hosts, in CF it is generally considered to be benign [43].

\section{Respiratory Viruses}

Many respiratory viruses are associated with CF exacerbations and decline in FEV1 [44]. Between $50 \%$ and $60 \%$ of pulmonary exacerbations have a viral component [45]. Children with an active pulmonary exacerbation were nearly three times as likely to have a viral infection compared to those near their baseline [46].

The biodiversity of viral infections varies with age and geographic location. The most common viral infections are respiratory syncytial virus (RSV), influenza A and $\mathrm{B}$ virus, parainfluenza virus, cytomegalovirus (CMV), human rhinovirus (HRV), human metapneumovirus (HMPV), coronavirus, and adenovirus [47]. Across the age spectrum, HRV is the most common viral infection. RSV is more common in younger patients and HMPV in older [48].

Pulmonary exacerbations occur more frequently in the winter months with the annual emergence of influenza and respiratory syncytial virus (RSV) (Fig. 8.3) [49]. 


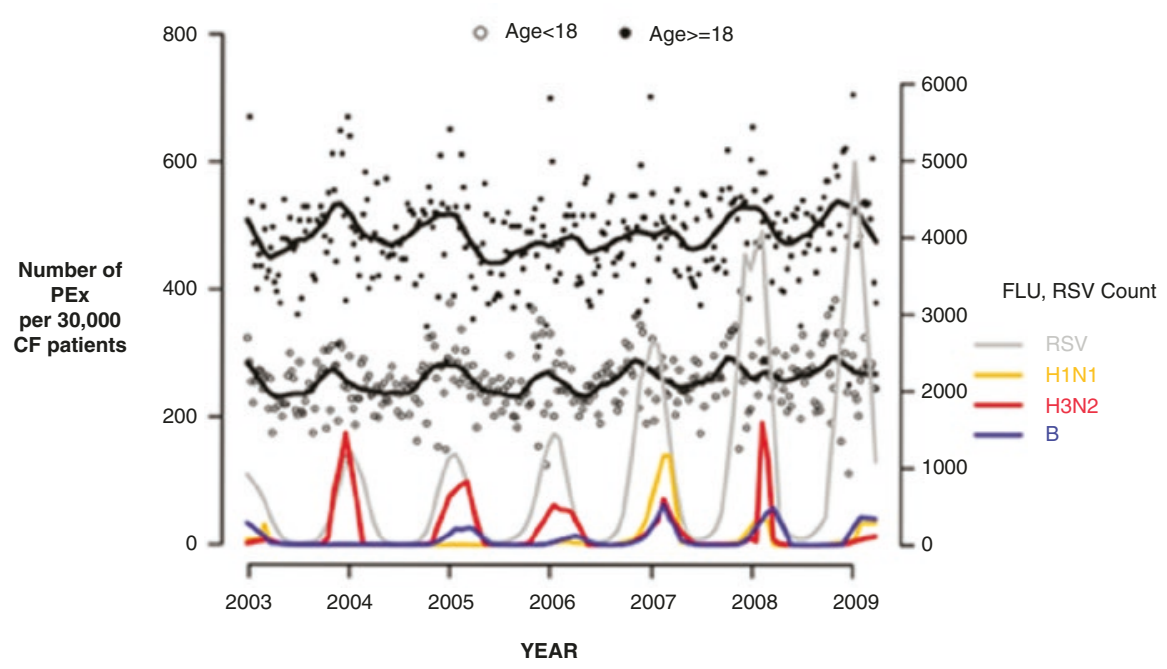

Fig. 8.3 Respiratory viruses in cystic fibrosis. Abbreviations: PEx pulmonary exacerbation, FLU influenza, RSV respiratory syncytial virus

Unlike bacterial infections, CF patients do not have an increased risk of viral infections; they have similar viral respiratory infections as their unaffected siblings [50]. However, when CF patients do contract a viral infection, they are more likely to develop a lower respiratory tract infection, be exposed to more antibiotics, and have a decline in FEV1 [51]. Viral infections such as influenza A and RSV promote bacterial adherence, enhancing biofilm formation and increasing the rate of lower respiratory tract infections [52].

Annual vaccination against influenza is strongly recommended and helps prevent the disease and its complications. During the 2009 H1N1 pandemic, CF patients in Europe who tested positive for influenza had a vaccination rate of only $8.8 \%$ whereas the overall vaccination rate in CF patients was estimated to be 75\% [53]. It is also recommended that high-risk CF patients (having four or more exacerbations per year) receive neuraminidase inhibitor prophylaxis during influenza season as this group has nearly 10 times the risk of a PEx during influenza season as lower risk CF patients [54].

RSV is an important pathogen in children although it does not seem to be associated with pulmonary exacerbations in adults [49]. RSV is associated with early pulmonary decline and seems to increase PA antibiotic resistance by enhancing biofilm production [1]. RSV infections also increase the risk of developing chronic PA infections and increase inflammation in patients already chronically infected with PA [55]. There has been much interest in preventing RSV infections pharmacologically in CF patients. However, palivizumab (a monoclonal antibody against RSV antigen) does not seem to be effective at decreasing RSV related morbidity in infants with CF who do not otherwise meet criteria for administration [56]. While there are RSV vaccines that are being evaluated, their benefit in patients with $\mathrm{CF}$ remains to be established [57]. 


\section{Pulmonary Exacerbations (PEx): Diagnosis and Etiology}

Pulmonary exacerbations decrease lung function, affect quality of life, and increase mortality [58]. Historically, there has been a lack of consensus on the definition of PEx [59]. In retrospective studies, PEx are often identified by the administration of intravenous antibiotics due to pulmonary symptoms. Many other criteria have been suggested. All include some combination of the following symptoms: an increase in sputum volume or color, new or increased hemoptysis, increased cough, increased dyspnea, malaise, fatigue, temperature $>38 \mathrm{C}$, anorexia/weight loss, sinus pain, change in sinus discharge, change in physical examination of the chest, decrease in pulmonary function by $\geq 10 \%$, or radiographic changes in support of a pulmonary infection $[1,60,61]$. According to the STOP trial, which gathered data on current practices across CF Foundation-accredited care centers, most clinicians also rely on an acute drop in lung function to diagnose a PEx [62]. There is no well-accepted method to further classify a PEx by its severity.

Many biomarkers have been considered as PEx diagnostic aids, but to date none have been performed acceptably well for clinical use. Procalcitonin, CRP, IL-18, and IL-6 have all been generally unhelpful in predicting an imminent exacerbation [63]. Calprotectin does demonstrate some early promise with some ability to predict PEx, but its clinical utility remains unclear [64]. Matouk et al. showed that patients with a higher hsCRP were more likely to have more clinically active disease and future PEx [65]. Biomarker panels may prove to be more useful than any single biomarker. One example, the MRM-MS protein panel which compiled six biomarkers, was better able to identify patients with imminent PEx than using FEV1 alone [66].

Pulmonary exacerbations can be triggered by infections, environmental exposures, or may be idiopathic. Acute viral infections are thought to contribute to about half of all exacerbations [46]. Chronic pulmonary infections with gram-negative organisms such as PA, BCC, or Stenotrophomonas maltophilia also place patients at a high risk for PEx without any other obvious precipitant [67]. It is not well understood how these chronic bacterial colonizers can lead to PEx. Bacterial overgrowth or new resistance patterns are likely possibilities [1].

\section{Pulmonary Exacerbations: Treatment}

Initial approaches to antimicrobial therapy are usually empiric, informed by prior sputum culture and antimicrobial history. Supplemental oxygenation, positive pressure ventilation, intravenous rehydration, and optimization of nutrition may also be necessary [68]. Unfortunately, best practices for many aspects of PEx management remain unclear. Guidelines cite insufficient evidence to make a firm recommendation regarding antibiotic route (ie. addition of inhaled to IV antibiotics), duration of antibiotics, site of treatment (inpatient vs. home), and therapies such as systemic 
steroids $[68,69]$. This ambiguity is well recognized by CF providers, and research is underway to bridge these gaps and develop more standardized care [62].

The decision to admit depends on the patient's clinical status as well as home resources. Home utilities, caretaker availability, and medication administration all must be ensured. Antibiotic monitoring and pulmonary therapies are often much more easily performed in the inpatient setting.

\section{Antibiotic Therapy}

Sputum samples often grow a multiplicity of organisms, and studies have not yet elucidated the best antibiotic approach when this occurs [70]. Furthermore, resistance patterns add another layer of biodiversity and may not be reflected in a single sputum sample. Nevertheless, isolates from previous patient encounters should assist in tailoring a patient-centered approach [67]. All treatment should be informed by both the local antibiogram and the patient's prior and current sputum cultures.

There are no recommendations regarding oral versus intravenous antibiotic therapy. Patients treated with intravenous antibiotics do have a faster improvement in FEV1 than patients treated with oral antibiotics, but they also are sicker - starting further below their baseline lung function [71]. European guidelines recommend starting with empiric oral antibiotics to cover common organisms including $H$. influenzae and Staphylococcus. Ciprofloxacin may be given if the patient has a prior history of Pseudomonas [60]. Despite the standard use of inhaled antibiotics for prevention of PEx, inhaled antibiotics are not equivalent to intravenous antibiotics for treatment of PEx [69].

The STOP study also found a large variation in length of antibiotic courses prescribed, which averaged 16 days and ranged from 2 to 51 days [62]. A minimum duration of 10 days has been recommended for antibiotic treatment based on a study from 2016 which found that patients treated for 9 days or less were at an increased risk of requiring retreatment with intravenous antibiotics in the next 30 days according to the CFF patient annual report. Notably, this was in patients treated in the outpatient setting only $[62,72]$. The ongoing STOP2 study plans to focus on treatment duration and its implications, weighing early relapse with FEV1 improvement and toxicity [58].

Methicillin-sensitive Staphylococcus aureus should be treated with narrow spectrum beta-lactams according to the local antibiogram. MRSA colonization should be treated during a PEx according to sensitivities [1, 62]. First-line therapy for MRSA in PEx is vancomycin or linezolid [70]. However, antibiotic choice should be individualized, and other options for therapy include trimethoprim-sulfamethoxazole, doxycycline, minocycline, clindamycin, levofloxacin, teicoplanin, and rifampin [62]. It should be noted that vancomycin does not penetrate biofilm, and vancomycin resistance also confers teicoplanin resistance [73]. Doxycycline should not typically be used in patients less than 8 years old. Linezolid has several potential side effects including serotonin syndrome, irreversible neuropathy, and severe lactic 
acidosis. Resistance to linezolid is increasing as it is used more frequently [73]. At our center, we often cover for MRSA with clindamycin, trimethoprimsulfamethoxazole, or vancomycin, depending on the patient's history.

Pseudomonas aeruginosa is a prevalent pathogen that contributes to a disproportionate amount of lung function decline and mortality [67]. Often, two antibiotics are used in the clinical setting against PA. The Cystic Fibrosis Foundation states there is insufficient evidence to support combination therapy for PA and that in certain situations, especially where the disease stage is mild, single therapy may be used [68]. When two antibiotics are chosen, they should have different mechanisms of action. The combination of a beta-lactam and an aminoglycoside such as tobramycin is frequently used [67]. Once-daily dosing for aminoglycosides is preferred [68]. The most commonly used beta-lactams in CF Foundation-accredited centers include ceftazidime, piperacillin-tazobactam, meropenem, and cefepime [74].

Burkholderia cepacia complex is resistant to aminoglycosides and frequently to beta-lactams as well [1]. Broad spectrum antibiotics including meropenem, piperacillin-tazobactam, aztreonam, and ceftazidime are often used first line [1, 60]. Tetracyclines, ciprofloxacin, and trimethoprim/sulfamethoxazole are also appropriate options [75]. Treatment should be tailored to current and past resistance $\mathrm{BCC}$ patterns. If significant resistance or cepacia syndrome is apparent, treatment requires multiple antibiotics simultaneously with more than one beta-lactam [1, 75]. There are some studies that suggest early treatment can prevent chronic BCC colonization [75].

Treatment of choice for Stenotrophomonas maltophilia is trimethoprimsulfamethoxazole plus ticarcillin-clavulanate, levofloxacin, doxycycline, or tigecycline [70]. European guidelines recommend cotrimoxazole as the drug of choice with alternatives including tetracyclines, ticarcillin-clavulanate, and tigecycline [60].

The primary treatment for viral-induced exacerbations is supportive care and monitoring for secondary bacterial infections. Neuraminidase inhibitors have been poorly studied in cystic fibrosis [76]. Although the efficacy of these medications is in question, the NICE guidelines do recommend that patients with chronic lung disease are treated for influenza [77].

The mainstay of treatment for allergic bronchopulmonary aspergillosis (ABPA) is corticosteroid therapy, usually prednisone at $0.5 \mathrm{mg} / \mathrm{kg} / \mathrm{d}$ for 2 weeks followed by a month-long taper. Higher doses of steroids had more adverse effects and were not more effective [78]. Additional therapy with antifungals and monoclonal antibodies against IgE may be necessary $[39,79]$.

Nontuberculous mycobacteria (NTM) are difficult to eradicate, requiring multidrug therapy for recommended treatment courses of 12 months or more. M. abscessus is notoriously difficult to treat with high rates of failure and recurrence despite optimal therapy [19]. Treatment of $M$. abscessus is divided into an initiation phase lasting 3-12 weeks and a continuation phase, which should continue for 12 months following three negative sputum cultures [19]. The initiation phase consists of azithromycin and intravenous amikacin, and one or more of the following: tigecycline, imipenem, or cefoxitin. Transition to the continuation phase is patient specific, depending on clinical response. The continuation phase consists of daily 
azithromycin, inhaled amikacin, and two or three of the following: minocycline, clofazimine, moxifloxacin, and/or linezolid.

M. avium treatment is usually successful with oral therapy - typically azithromycin plus rifampin and ethambutol. Macrolide susceptibility should be tested prior to starting treatment for M. avium and in resistant or recurrent cases.

Although antibiotics are the cornerstone of treatment for a PEx, they are only one piece of the care that should be received. Respiratory clearance treatments and chest physiotherapy are crucial for functional improvement. Glucose management is also important, especially as insulin requirements can increase greatly during these physiological stressful periods and during corticosteroid therapy [67]. Steroid use in treatment of PEx, without a specific indication such as with ABPA, has had varying results in the literature and is, therefore, not currently recommended by the CFF [68]. Nutritional optimization is important as low BMI is associated with worsened prognosis and infections increase catabolism.

\section{Chronic Infections}

All patients 6 years and older should undergo regular surveillance with sputum cultures and sensitivities quarterly and NTM cultures annually [80].

While MRSA presence confers worse clinical outcomes in CF patients, optimal management remains unclear. The STAR-TOO trial found that eradication of new MRSA infections was feasible for most (not all) newly infected CF patients, which may suggest a long-term clinical benefit. Their treatment protocol included oral antibiotics, decolonization (with nasal mupirocin, chlorhexidine body and mouth washes), and enhanced household cleaning [81]. However, in persistently infected CF patients, MRSA eradication rates remained poor even with the addition of inhaled vancomycin in the Persistent MRSA Eradication Protocol (PMEP) trial. Furthermore, inhaled vancomycin caused intolerable bronchospasms in some patients [82].

While the US guidelines await further evidence for benefit, the European Cystic Fibrosis Trust recommends aiming for MRSA eradication in newly infected patients with nasal mupirocin, chlorhexidine bath wash, and either combination oral therapy with rifampicin and fusidic acid (not available in the US), or nebulized vancomycin, or a combination of all three [83].

If patients become infected with PA, early eradication should be attempted. Current guidelines recommend inhaled tobramycin $300 \mathrm{mg}$ BID for 28 days to attempt eradication [13]. Twenty-eight days of inhaled aztreonam also seems to be an effective eradication strategy for newly acquired PA [84]. Inhaled colistin and oral ciprofloxacin are first-line options in the United Kingdom for initial colonization [83]. Chronic colonization of PA is defined as three positive cultures each a month apart or more [13]. Ongoing inhaled aztreonam and tobramycin are recommended for treatment of these patients, typically administered in a 1-month on, 1-month off pattern [17]. Lastly, there is evidence that ivacaftor has some anti-PA and anti-SA 

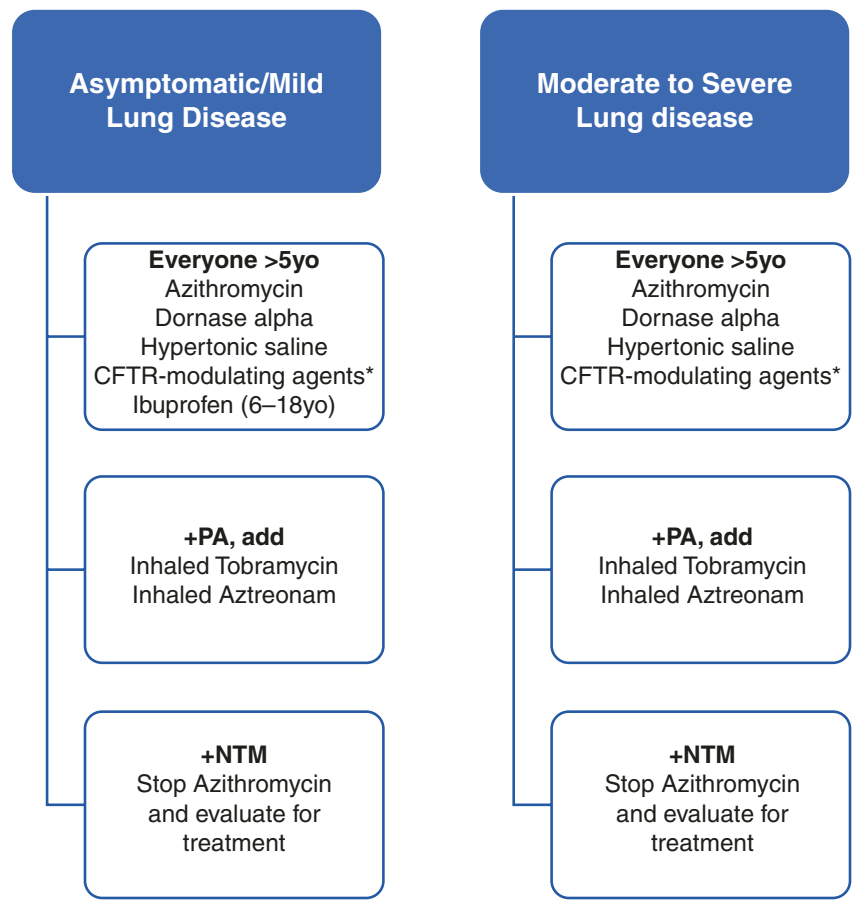

Don't use:

Inhaled corticosteroids (exceptions: Asthma and $\mathrm{ABPA}$ )

Oral corticosteroids

Fig. 8.4 Recommendations for chronic pulmonary therapies based on severity of lung disease for CF patients $>5$ year old

activity due to its quinolone-like structure, explaining another mechanism for the drug's efficacy beyond the CFTR modulation [85].

Current guidelines recommend chronic azithromycin for all $\mathrm{CF}$ patients older than 6 years who are chronically infected with Pseudomonas due to a significant reduction in PEx [17]. In those not infected with PA, chronic azithromycin can be used for the same purpose, although evidence for this is less robust [17]. One concern is that chronic azithromycin use may lead to resistance or partial treatment if NTM is present. This reinforces the need for NTM screening at least annually (Fig. 8.4).

\section{Pharmacokinetic Considerations}

CF patients generally require larger loading doses of antibiotics compared to the general population [1]. Some suspect this is due to lower protein binding and alterations in the volume of distribution due to chronic malnutrition [86]. Two commonly classes of antibiotics prescribed during a PEx, antipseudomonal beta-lactams and aminoglycosides, have interesting pharmacokinetic implications [74]. 
Although beta-lactam serum drug levels are not routinely measured, significant changes in antibiotic clearance can occur during PEx. An average increased clearance of $20 \%$ results in beta-lactam levels being therapeutic only $45 \%$ of the time, which seems to have implications on airway microbiome $[87,88]$. However, the CF Foundation states in their guidelines that there is little evidence to support continuous infusion of beta-lactam antibiotics [68].

Intravenous aminoglycosides dosed once daily is as effective as thrice daily and is associated with less renal toxicity [89]. It is standard practice in pediatric CF Foundation-accredited care centers and affiliate programs to monitor aminoglycoside serum concentrations during PEx [90]. Tobramycin is typically given at $10 \mathrm{mg} /$ $\mathrm{kg}$ daily and is initially monitored with two random levels to calculate the maximum concentration (Cmax), with a goal Cmax of 20-40 mg/L [74]. A trough level is drawn 1 week after therapeutic dose attainment [90]. Further periodic monitoring is crucial as tobramycin clearance rates decrease by an average of $6 \%$ during PEx, and creeping levels can lead to nephrotoxicity and ototoxicity [91, 92].

\section{Lung Transplantation}

Bilateral lung transplantation is, in a sense, curative for the pulmonary manifestations of cystic fibrosis; donor lungs have functioning CFTR receptors and have not sustained the chronic anatomic injury of $\mathrm{CF}$ such as bronchiectasis. However, patients continue to have unique infectious risks following transplantation. Preexisting organisms tend to find their way into the transplanted lungs, likely harboring in nontransplanted airway space such as the sinuses. Most of these organisms such as PA tend to be easily treated. A few, especially Burkholderia cenocepacia, are associated with reduced survival and may be considered contraindications to transplant reception [93].

Lung transplantation requires life-long immunosuppression, which carries unique infectious disease considerations which are outside the scope of this text.

\section{Infection Prevention and Control}

Patients with CF can acquire infections through a variety of means. Direct contact with infectious secretions can be easily avoided. Indirect contact can also occur via fomites such as stethoscopes or contaminated surfaces; these objects should be disinfected regularly. Nebulizers and other potentially shared devices should be reserved for single-patient use only. Respiratory viruses can readily travel from person to person by infectious droplet nuclei which can spread as far as 6 feet.

While respiratory bacterial pathogens are not usually passed from healthy person to healthy person, transmission between patients who both have CF is increasingly recognized. Therefore, all effort should be taken to minimize close interactions 
between patients with CF. They should never share a room unless already living within the same household. Clinics should optimize flow to minimize patients' time in waiting rooms and other common areas. Rather than rotate $\mathrm{CF}$ patients through various areas of a clinic, staff members should rotate into the patient's singular room. CF patients should wear a surgical mask whenever in shared areas of the healthcare setting, such as hallways and restrooms. However, they do not need to wear a mask in their own clinic or hospital exam room.

Healthcare providers should wear a gown and gloves whenever interacting with patients with $\mathrm{CF}$, whether inpatient or outpatient, regardless of recent culture results. Providers may also need to wear an appropriate mask, but only if droplet or airborne precautions are otherwise indicated based on the suspected or confirmed presence of infectious pathogens (i.e., adenovirus, mycobacteria, etc).

Patients and providers alike should always practice meticulous and frequent hand hygiene [94].

\section{References}

1. Uluer A, Marty F. Cystic fibrosis. In: Blaser M, Dolin R, Bennett J, editors. Principles and practices of infectious disease. 1. 8th ed. Philadelphia: Saunders; 2014. p. 874-85.

2. Lipuma JJ. The changing microbial epidemiology in cystic fibrosis. Clin Microbiol Rev. 2010;23(2):299-323.

3. Grody WW, Cutting GR, Klinger KW, Richards CS, Watson MS, Desnick RJ. Laboratory standards and guidelines for population-based cystic fibrosis carrier screening. Genet Med. 2001;3(2):149-54.

4. Gabriel SE, Brigman KN, Koller BH, Boucher RC, Stutts MJ. Cystic fibrosis heterozygote resistance to cholera toxin in the cystic fibrosis mouse model. Science. 1994;266(5182):107-9.

5. Cystic Fibrosis Foundation Patient Registry. 2017 annual data report. Bethesda; 2017.

6. Rosenfeld M, Bernardo-Ocampo C, Emerson J, Genatossio A, Burns J, Gibson R. Prevalence of cystic fibrosis pathogens in the oropharynx of healthy children and implications for cystic fibrosis care. J Cyst Fibros. 2012;11(5):456-7.

7. Dasenbrook EC, Checkley W, Merlo CA, Konstan MW, Lechtzin N, Boyle MP. Association between respiratory tract methicillin-resistant Staphylococcus aureus and survival in cystic fibrosis. JAMA. 2010;303(23):2386-92.

8. Wilmott RW, Tyson SL, Matthew DJ. Cystic fibrosis survival rates. The influences of allergy and Pseudomonas aeruginosa. Am J Dis Child. 1985;139(7):669-71.

9. Saiman L, Siegel JD, LiPuma JJ, Brown RF, Bryson EA, Chambers MJ, et al. Infection prevention and control guideline for cystic fibrosis: 2013 update. Infect Control Hosp Epidemiol. 2014;35(Suppl 1):S1-S67.

10. Cramer N, Wiehlmann L, Tummler B. Clonal epidemiology of Pseudomonas aeruginosa in cystic fibrosis. Int J Med Microbiol. 2010;300(8):526-33.

11. Hoiby N, Ciofu O, Bjarnsholt T. Pseudomonas aeruginosa biofilms in cystic fibrosis. Future Microbiol. 2010;5(11):1663-74.

12. Lee TW, Brownlee KG, Conway SP, Denton M, Littlewood JM. Evaluation of a new definition for chronic Pseudomonas aeruginosa infection in cystic fibrosis patients. J Cyst Fibros. 2003;2(1):29-34.

13. Mogayzel PJ Jr, Naureckas ET, Robinson KA, Brady C, Guill M, Lahiri T, et al. Cystic Fibrosis Foundation pulmonary guideline. Pharmacologic approaches to prevention and eradication of initial Pseudomonas aeruginosa infection. Ann Am Thorac Soc. 2014;11(10):1640-50. 
14. Hutchinson J, Runge W, Mulvey M, Norris G, Yetman M, Valkova N, et al. Burkholderia cepacia infections associated with intrinsically contaminated ultrasound gel: the role of microbial degradation of parabens. Infect Control Hosp Epidemiol. 2004;25(4):291-6.

15. Courtney JM, Dunbar KE, McDowell A, Moore JE, Warke TJ, Stevenson M, et al. Clinical outcome of Burkholderia cepacia complex infection in cystic fibrosis adults. J Cyst Fibros. 2004;3(2):93-8.

16. Kalish LA, Waltz DA, Dovey M, Potter-Bynoe G, McAdam AJ, Lipuma JJ, et al. Impact of Burkholderia dolosa on lung function and survival in cystic fibrosis. Am J Respir Crit Care Med. 2006;173(4):421-5.

17. Mogayzel PJ Jr, et al. Cystic fibrosis pulmonary guidelines. Chronic medications for maintenance of lung health. Am J Respir Crit Care Med. 2013;187(7):680-9.

18. Olivier KN, Weber DJ, Wallace RJ Jr, Faiz AR, Lee JH, Zhang Y, et al. Nontuberculous mycobacteria. I: multicenter prevalence study in cystic fibrosis. Am J Respir Crit Care Med. 2003;167(6):828-34.

19. Floto RA, Olivier KN, Saiman L, Daley CL, Herrmann JL, Nick JA, et al. US Cystic Fibrosis Foundation and European Cystic Fibrosis Society consensus recommendations for the management of non-tuberculous mycobacteria in individuals with cystic fibrosis. Thorax. 2016;71(Suppl 1):i1-22.

20. Cavalli Z, Reynaud Q, Bricca R, Nove-Josserand R, Durupt S, Reix P, et al. High incidence of non-tuberculous mycobacteria-positive cultures among adolescent with cystic fibrosis. J Cyst Fibros. 2017;16(5):579-84.

21. Qvist T, Gilljam M, Jonsson B, Taylor-Robinson D, Jensen-Fangel S, Wang M, et al. Epidemiology of nontuberculous mycobacteria among patients with cystic fibrosis in Scandinavia. J Cyst Fibros. 2015;14(1):46-52.

22. Park IK, Olivier KN. Nontuberculous mycobacteria in cystic fibrosis and non-cystic fibrosis bronchiectasis. Semin Respir Crit Care Med. 2015;36(2):217-24.

23. Long S, Prober C, Fischer M, editors. Principles and practice of pediatric infectious diseases. 5th ed. Philadelphia: Elsevier Health Sciences; c2018.

24. Waters V, Atenafu EG, Lu A, Yau Y, Tullis E, Ratjen F. Chronic Stenotrophomonas maltophilia infection and mortality or lung transplantation in cystic fibrosis patients. J Cyst Fibros. 2013;12(5):482-6.

25. Goss CH, Mayer-Hamblett N, Aitken ML, Rubenfeld GD, Ramsey BW. Association between Stenotrophomonas maltophilia and lung function in cystic fibrosis. Thorax. 2004;59(11):955-9.

26. Waters V, Yau Y, Prasad S, Lu A, Atenafu E, Crandall I, et al. Stenotrophomonas maltophilia in cystic fibrosis: serologic response and effect on lung disease. Am J Respir Crit Care Med. 2011;183(5):635-40.

27. Hansen CR, Pressler T, Nielsen KG, Jensen PO, Bjarnsholt T, Hoiby N. Inflammation in Achromobacter xylosoxidans infected cystic fibrosis patients. J Cyst Fibros. 2010;9(1):51-8.

28. Ronne Hansen C, Pressler T, Hoiby N, Gormsen M. Chronic infection with Achromobacter xylosoxidans in cystic fibrosis patients; a retrospective case control study. J Cyst Fibros. 2006;5(4):245-51.

29. De Baets F, Schelstraete P, Van Daele S, Haerynck F, Vaneechoutte M. Achromobacter xylosoxidans in cystic fibrosis: prevalence and clinical relevance. J Cyst Fibros. 2007;6(1):75-8.

30. Lambiase A, Catania MR, Del Pezzo M, Rossano F, Terlizzi V, Sepe A, et al. Achromobacter xylosoxidans respiratory tract infection in cystic fibrosis patients. Eur J Clin Microbiol Infect Dis. 2011;30(8):973-80.

31. Flight WG, Smith A, Paisey C, Marchesi JR, Bull MJ, Norville PJ, et al. Rapid detection of emerging pathogens and loss of microbial diversity associated with severe lung disease in cystic fibrosis. J Clin Microbiol. 2015;53(7):2022-9.

32. Tunney MM, Field TR, Moriarty TF, Patrick S, Doering G, Muhlebach MS, et al. Detection of anaerobic bacteria in high numbers in sputum from patients with cystic fibrosis. Am J Respir Crit Care Med. 2008;177(9):995-1001. 
33. Liu JC, Modha DE, Gaillard EA. What is the clinical significance of filamentous fungi positive sputum cultures in patients with cystic fibrosis? J Cyst Fibros. 2013;12(3):187-93.

34. Schwarz C, Hartl D, Eickmeier O, Hector A, Benden C, Durieu I, et al. Progress in definition, prevention and treatment of fungal infections in cystic fibrosis. Mycopathologia. 2018;183(1):21-32.

35. Maturu VN, Agarwal R. Prevalence of Aspergillus sensitization and allergic bronchopulmonary aspergillosis in cystic fibrosis: systematic review and meta-analysis. Clin Exp Allergy. 2015;45(12):1765-78.

36. Bakare N, Rickerts V, Bargon J, Just-Nubling G. Prevalence of Aspergillus fumigatus and other fungal species in the sputum of adult patients with cystic fibrosis. Mycoses. 2003;46(1-2):19-23.

37. de Vrankrijker AM, van der Ent CK, van Berkhout FT, Stellato RK, Willems RJ, Bonten MJ, et al. Aspergillus fumigatus colonization in cystic fibrosis: implications for lung function? Clin Microbiol Infect. 2011;17(9):1381-6.

38. Baxter CG, Moore CB, Jones AM, Webb AK, Denning DW. IgE-mediated immune responses and airway detection of Aspergillus and Candida in adult cystic fibrosis. Chest. 2013;143(5):1351-7.

39. Stevens DA, Moss RB, Kurup VP, Knutsen AP, Greenberger P, Judson MA, et al. Allergic bronchopulmonary aspergillosis in cystic fibrosis - state of the art: Cystic Fibrosis Foundation Consensus Conference. Clin Infect Dis. 2003;37(Suppl 3):S225-64.

40. Valenza G, Tappe D, Turnwald D, Frosch M, Konig C, Hebestreit H, et al. Prevalence and antimicrobial susceptibility of microorganisms isolated from sputa of patients with cystic fibrosis. J Cyst Fibros. 2008;7(2):123-7.

41. Gileles-Hillel A, Shoseyov D, Polacheck I, Korem M, Kerem E, Cohen-Cymberknoh M. Association of chronic Candida albicans respiratory infection with a more severe lung disease in patients with cystic fibrosis. Pediatr Pulmonol. 2015;50(11):1082-9.

42. Sahi H, Avery RK, Minai OA, Hall G, Mehta AC, Raina P, et al. Scedosporium apiospermum (Pseudoallescheria boydii) infection in lung transplant recipients. J Heart Lung Transplant. 2007;26(4):350-6.

43. Kondori N, Gilljam M, Lindblad A, Jonsson B, Moore ER, Wenneras C. High rate of Exophiala dermatitidis recovery in the airways of patients with cystic fibrosis is associated with pancreatic insufficiency. J Clin Microbiol. 2011;49(3):1004-9.

44. Smyth AR, Smyth RL, Tong CY, Hart CA, Heaf DP. Effect of respiratory virus infections including rhinovirus on clinical status in cystic fibrosis. Arch Dis Child. 1995;73(2):117-20.

45. Asner S, Waters V, Solomon M, Yau Y, Richardson SE, Grasemann H, et al. Role of respiratory viruses in pulmonary exacerbations in children with cystic fibrosis. J Cyst Fibros. 2012;11(5):433-9.

46. Wat D, Gelder C, Hibbitts S, Cafferty F, Bowler I, Pierrepoint M, et al. The role of respiratory viruses in cystic fibrosis. J Cyst Fibros. 2008;7(4):320-8.

47. Ong EL, Ellis ME, Webb AK, Neal KR, Dodd M, Caul EO, et al. Infective respiratory exacerbations in young adults with cystic fibrosis: role of viruses and atypical microorganisms. Thorax. 1989;44(9):739-42.

48. Flight WG, Bright-Thomas RJ, Tilston P, Mutton KJ, Guiver M, Morris J, et al. Incidence and clinical impact of respiratory viruses in adults with cystic fibrosis. Thorax. 2014;69(3):247-53.

49. Somayaji R, Goss CH, Khan U, Neradilek M, Neuzil KM, Ortiz JR. Cystic fibrosis pulmonary exacerbations attributable to respiratory syncytial virus and influenza: a population-based study. Clin Infect Dis. 2017;64(12):1760-7.

50. Ramsey BW, Gore EJ, Smith AL, Cooney MK, Redding GJ, Foy H. The effect of respiratory viral infections on patients with cystic fibrosis. Am J Dis Child. 1989;143(6):662-8.

51. Hiatt PW, Grace SC, Kozinetz CA, Raboudi SH, Treece DG, Taber LH, et al. Effects of viral lower respiratory tract infection on lung function in infants with cystic fibrosis. Pediatrics. 1999;103(3):619-26. 
52. Hament JM, Kimpen JL, Fleer A, Wolfs TF. Respiratory viral infection predisposing for bacterial disease: a concise review. FEMS Immunol Med Microbiol. 1999;26(3-4):189-95.

53. Viviani L, Assael BM, Kerem E, Group EHNs. Impact of the A (H1N1) pandemic influenza (season 2009-2010) on patients with cystic fibrosis. J Cyst Fibros. 2011;10(5):370-6.

54. Ortiz JR, Neuzil KM, Victor JC, Wald A, Aitken ML, Goss CH. Influenza-associated cystic fibrosis pulmonary exacerbations. Chest. 2010;137(4):852-60.

55. Petersen NT, Hoiby N, Mordhorst CH, Lind K, Flensborg EW, Bruun B. Respiratory infections in cystic fibrosis patients caused by virus, chlamydia and mycoplasma--possible synergism with Pseudomonas aeruginosa. Acta Paediatr Scand. 1981;70(5):623-8.

56. Robinson KA, Odelola OA, Saldanha IJ. Palivizumab for prophylaxis against respiratory syncytial virus infection in children with cystic fibrosis. Cochrane Database Syst Rev. 2016;7:CD007743.

57. Graham CS. Vaccine development for respiratory syncytial virus. Curr Opin Virol. 2017;23:107-12.

58. Heltshe SL, West NE, VanDevanter DR, Sanders DB, Beckett VV, Flume PA, et al. Study design considerations for the standardized treatment of pulmonary exacerbations 2 (STOP2): a trial to compare intravenous antibiotic treatment durations in CF. Contemp Clin Trials. 2018;64:35-40.

59. Rosenfeld M, Emerson J, Williams-Warren J, Pepe M, Smith A, Montgomery AB, et al. Defining a pulmonary exacerbation in cystic fibrosis. J Pediatr. 2001;139(3):359-65.

60. Bhatt JM. Treatment of pulmonary exacerbations in cystic fibrosis. Eur Respir Rev. 2013;22(129):205-16.

61. Goss CH, Burns JL. Exacerbations in cystic fibrosis. 1: epidemiology and pathogenesis. Thorax. 2007;62(4):360-7.

62. West NE, Beckett VV, Jain R, Sanders DB, Nick JA, Heltshe SL, et al. Standardized treatment of pulmonary exacerbations (STOP) study: physician treatment practices and outcomes for individuals with cystic fibrosis with pulmonary exacerbations. J Cyst Fibros. 2017;16(5):600-6.

63. Scott LK, Toner R. Clinically promising biomarkers in cystic fibrosis pulmonary exacerbations. Lung. 2017;195(4):397-401.

64. Gray RD, Downey D, Taggart CC. Biomarkers to monitor exacerbations in cystic fibrosis. Expert Rev Respir Med. 2017;11(4):255-7.

65. Matouk E, Nguyen D, Benedetti A, Bernier J, Gruber J, Landry J, et al. C-reactive protein in stable cystic fibrosis: an additional Indicator of clinical disease activity and risk of future pulmonary exacerbations. J Pulm Respir Med. 2016;6(5):1000375.

66. Quon BS, Dai DL, Hollander Z, Ng RT, Tebbutt SJ, Man SF, et al. Discovery of novel plasma protein biomarkers to predict imminent cystic fibrosis pulmonary exacerbations using multiple reaction monitoring mass spectrometry. Thorax. 2016;71(3):216-22.

67. Smyth A, Elborn JS. Exacerbations in cystic fibrosis: 3--management. Thorax. 2008;63(2):180-4.

68. Flume PA, et al. Cystic fibrosis pulmonary guidelines: treatment of pulmonary exacerbations. Am J Respir Crit Care Med. 2009;180(9):802-8.

69. Hurley MN, Prayle AP, Flume P. Intravenous antibiotics for pulmonary exacerbations in people with cystic fibrosis. Paediatr Respir Rev. 2015;16(4):246-8.

70. Chmiel JF, Aksamit TR, Chotirmall SH, Dasenbrook EC, Elborn JS, LiPuma JJ, et al. Antibiotic management of lung infections in cystic fibrosis. I. The microbiome, methicillinresistant Staphylococcus aureus, gram-negative bacteria, and multiple infections. Ann Am Thorac Soc. 2014;11(7):1120-9.

71. Wagener JS, et al. Oral, inhaled, and intravenous antibiotic choice for treating pulmonary exacerbations in cystic fibrosis. Pediatr Pulmonol. 2013;48(7):666-73.

72. VanDevanter DR, Flume PA, Morris N, Konstan MW. Probability of IV antibiotic retreatment within thirty days is associated with duration and location of IV antibiotic treatment for pulmonary exacerbation in cystic fibrosis. J Cyst Fibros. 2016;15(6):783-90.

73. Goss CH, Muhlebach MS. Review: Staphylococcus aureus and MRSA in cystic fibrosis. J Cyst Fibros. 2011;10(5):298-306. 
74. Zobell JT, et al. Tobramycin and Beta-lactam antibiotic use in cystic fibrosis exacerbations: a pharmacist approach. J Pediatr Pharmacol Ther. 2016;21(3):239-46.

75. Horsley A, Webb K, Bright-Thomas R, Govan J, Jones A. Can early Burkholderia cepacia complex infection in cystic fibrosis be eradicated with antibiotic therapy? Front Cell Infect Microbiol. 2011;1:18. Published 2011 Dec 21. https://doi.org/10.3389/fcimb.2011.00018.

76. Jagannath VA, Asokan GV, Fedorowicz Z, Lee TW. Neuraminidase inhibitors for the treatment of influenza infection in people with cystic fibrosis. Cochrane Database Syst Rev. 2016;2:CD008139.

77. National Institute for Health and Care Excellence. Cystic fibrosis: diagnosis and management. Published Oct 2017. Available at: https://www.nice.org.uk/guidance/ng78.

78. Agarwal R. A randomised trial of glucocorticoids in acute-stage allergic bronchopulmonary aspergillosis complicating asthma. Eur Respir J. 2016;47(2):490-8.

79. Wong R, Wong M, Robinson PD, Fitzgerald DA. Omalizumab in the management of steroid dependent allergic bronchopulmonary aspergillosis (ABPA) complicating cystic fibrosis. Paediatr Respir Rev. 2018;14(1):22-4.

80. Yankaskas JR, Marshall BC, Sufian B, Simon RH, Rodman D. Cystic fibrosis adult care: consensus conference report. Chest. 2004;125(1 Suppl):1S-39S.

81. Muhlebach MS, Beckett V, Popowich E, Miller MB, Baines A, Mayer-Hamblett N, et al. Microbiological efficacy of early MRSA treatment in cystic fibrosis in a randomised controlled trial. Thorax. 2017;72(4):318-26.

82. Dezube R, Jennings MT, Rykiel M, Diener-West M, Boyle MP, Chmiel JF, et al. Eradication of persistent methicillin-resistant Staphylococcus aureus infection in cystic fibrosis. J Cyst Fibros. 2019;18(3):357-63.

83. Group. RotUCFTAW. Antibiotic treatment for cystic fibrosis. 3rd ed. Cystic Fibrosis Trust UK; 2009. Available from: http://www.cftrust.org.uk/aboutcf/publications/consensusdoc/ Antibiotic_treatment_for_Cystic_Fibrosis.pdf.

84. Tiddens HA, et al. Open label study of inhaled aztreonam for Pseudomonas eradication in children with cystic fibrosis: the ALPINE study. J Cyst Fibros. 2015;14(1):111-9.

85. Reznikov LR, Abou Alaiwa MH, Dohrn CL, Gansemer ND, Diekema DJ, Stoltz DA, et al. Antibacterial properties of the CFTR potentiator ivacaftor. J Cyst Fibros. 2014;13(5):515-9.

86. Bulitta JB, et al. Four decades of $\beta$-lactam antibiotic pharmacokinetics in cystic fibrosis. Clin Pharmacokinet. 2019;58(2):143-56.

87. Hong LT, Liou TG, Deka R, King JB, Steves V, Young DC. Pharmacokinetics of continuous infusion Beta-lactams in the treatment of acute pulmonary exacerbations in adult patients with cystic fibrosis. Chest. 2018;154(5):1108-14.

88. Hahn A, et al. Changes in microbiome diversity following beta-lactam antibiotic treatment are associated with therapeutic versus subtherapeutic antibiotic exposure in cystic fibrosis. Sci Rep. 2019;9(1):2534.

89. Smyth AR, Bhatt J, Nevitt SJ. Once-daily versus multiple-daily dosing with intravenous aminoglycosides for cystic fibrosis. Cochrane Database Syst Rev. 2017;3:CD002009.

90. Prescott WA Jr. National survey of extended-interval aminoglycoside dosing in pediatric cystic fibrosis pulmonary exacerbations. J Pediatr Pharmacol Ther. 2011;16(4):262-9.

91. Hong LT, Liou TG, Deka R, King JB, Stevens V, Young DC. Pharmacokinetics of continuous infusion Beta-lactams in the treatment of acute pulmonary exacerbations in adult patients with cystic fibrosis. Chest. 2018;154(5):1108-14.

92. Prayle A, Watson A, Fortnum H, Smyth A. Side effects of aminoglycosides on the kidney, ear and balance in cystic fibrosis. Thorax. 2010;65(7):654-8.

93. Dupont L. Lung transplantation in cystic fibrosis patients with difficult to treat lung infections. Curr Opin Pulm Med. 2017;23(6):574-9.

94. Saiman L, et al. Infection prevention and control guideline for cystic fibrosis: 2013 update. Infect Control Hosp Epidemiol. 2014;35(Suppl 1):S1-S67. 